\title{
Diagnosing Dry Eye Disease: Simplifying the Process and Avoiding Pitfalls
}

\author{
Marc Labetoulle ${ }^{1}$, Elisabeth M Messmer ${ }^{2}$ and Stefano Barabino ${ }^{3 *}$ \\ ${ }^{1}$ Head, Ophthalmology Department, South Paris University, Hôpitaux Universitaires \\ Paris Saclay, Kremlin-Bicêtre, France \\ ${ }^{2}$ Professor, Department of Ophthalmology, University of Munich, Munich, Germany \\ ${ }^{3}$ Head, Ocular Surface and Dry Eye Center, Ospedale L. Sacco, University of Milan, \\ Milan, Italy \\ *Corresponding Author: Stefano Barabino, Head, Ocular Surface and Dry Eye \\ Center, Ospedale L. Sacco, University of Milan, Milan, Italy.
}

Received: January 21, 2021

Published: February 08, 2021

(C) All rights are reserved by Stefano

Barabino., et al.

\begin{abstract}
Dry eye disease is often under diagnosed or misdiagnosed, in spite of its high prevalence and its impact on quality of life and mental health. The 2017 Definition and Classification report, the Tear Film and Ocular Surface Society (TFOS) Dry Eye Workshop (DEWS) II in 2017, continued to recognize dry eye disease as multifactorial, and updated the definition to include loss of homeostasis and neurosensory abnormalities as key elements of the disease. This review proposes key steps for simplifying and clarifying the diagnosis of dry eye disease in everyday clinical practice, based on the TFOS-DEWS-II report and the clinical experience of the ophthalmologist authors. It addresses key diagnostic principles, assessment of patient symptoms (such as dryness and ocular fatigue), and signs (such as tear film instability and meibomian dysfunction), the role of new technologies, and the potential pitfalls to avoid. Dry eye disease is a disease of the ocular system as a whole, and diagnosis can be an easy process if carried out systematically and logically. Fluorescein and lissamine green staining, and corneal sensitivity testing are simple tools that can provide a lot of diagnostic information. New technologies are also available which, although not essential for daily practice, can help explore the disease pathophysiology and also help communication with patients.
\end{abstract}

Keywords: Dry Eye Disease; Diagnosis; TFOS-DEWS II; Ocular Surface

\section{Abbreviations}

DEQ-5: Dry Eye Questionnaire-5; DEWS: Dry Eye Workshop; FBUT: Fluorescein Breakup Time; MGD: Meibomian Gland Dysfunction; MMP: Matrix Metalloproteinase; NBUT: Non-Invasive Breakup Time; NSAID: Non-Steroidal Anti-Inflammatory Drugs; OSDI: Ocular Surface Disease Index; OSD-QoL: Ocular Surface Disease-Quality of Life; TBUT: Tear Breakup Time; TFOS: Tear Film and Ocular Surface Society; TMH: Tear Meniscus Height; SANDE: Symptom Assessment in Dry Eye.

\section{Background}

Dry eye disease causes discomfort, pain, altered visual acuity, and has a significant impact on daily life, affecting activities such as reading, television viewing, driving, and working [1,2]. It is also associated with sleep disorders and interrupted sleep [3,4]. Symptoms of dry eye disease can negatively affect a patient's quality of life [1,5], and in some people the severity and chronicity of dry eye disease can lead to mood changes, anxiety and depression $[6,7]$. However, in spite of its widespread prevalence and impact, dry eye disease is often underdiagnosed or misdiagnosed $[1,8]$. In this review, we propose key steps for simplifying and clarifying the diagnosis of dry eye disease, based on clinical experience, and taking into account recent changes according to the Tear Film and Ocular Surface Society (TFOS) Dry Eye Workshop (DEWS) II definition and classification of dry eye disease [9]. Topics addressed will include key diagnostic principles, assessment of patient symptoms 
and signs, the role of new technologies and the potential pitfalls to avoid.

\section{Updated definition of dry eye disease}

In the 2017 TFOS DEWS II definition and classification report, dry eye disease is recognized as a multifactorial disease, with loss of homeostasis of the tear film as the central concept. Other key terms in the definition are tear film instability, hyperosmolarity, ocular surface inflammation and damage, and neurosensory abnormalities. The aim was to create an evidence-based definition and up to date classification system [9]. Three aspects of the revised definition are of note. The first two, homeostasis and neurosensory abnormalities, are new additions. Homeostasis is the multitude of intrinsic and extrinsic factors that are usually in balance with each other to maintain healthy ocular surface and normal tears. However, in dry eye disease, a loss of homeostasis means that these factors are imbalanced, and this is an important element in the pathogenesis of the disease. Neurosensory abnormalities are recognized as a new pathogenetic factor in the development of dry eye disease, due to an increase in evidence over the past 10 years or so. The abnormalities are related to corneal nerve damage, reduced corneal sensitivity, and ocular surface pain, but their exact role in the pathophysiological pathway of dry eye disease is not fully characterized [9].

The third aspect is something that could be added to complete the description of the conditions of DED: quality of vision. Indeed, the previous definition from 2007 included "visual disturbance" [9], a point that is very important when communicating with patients and authorities. It helps to clarify that dry eye disease is not just a nuisance or a discomfort, but that it can be accompanied by a decrease in functional visual acuity and vision loss.

\section{Diagnosing dry eye disease}

The following are commentaries on the key diagnostic steps proposed in the TFOS DEWS II publication, aiming to focus on the practical aspects of implementing their recommendations. Figure 1 shows the three diagnostic steps that are part of the overall management recommendations for dry eye disease - triaging questions, risk factors analysis and diagnostic tests [11].

Signs and the symptoms are not always in concordance in patients

A key point to appreciate when assessing patients for dry eye disease is that the symptoms, such as dryness, soreness and ocular fatigue, frequently do not concord with the clinical signs, such as ocular surface damage, tear film instability, and meibomian gland dysfunction [1,2,12-14]. An important factor here is corneal sensitivity, so a corneal sensitivity measurement should probably be added to the diagnostic procedure. Introducing the concept of neurotrophic or neuropathic keratopathy to the assessment is also important in patients with dry eye.

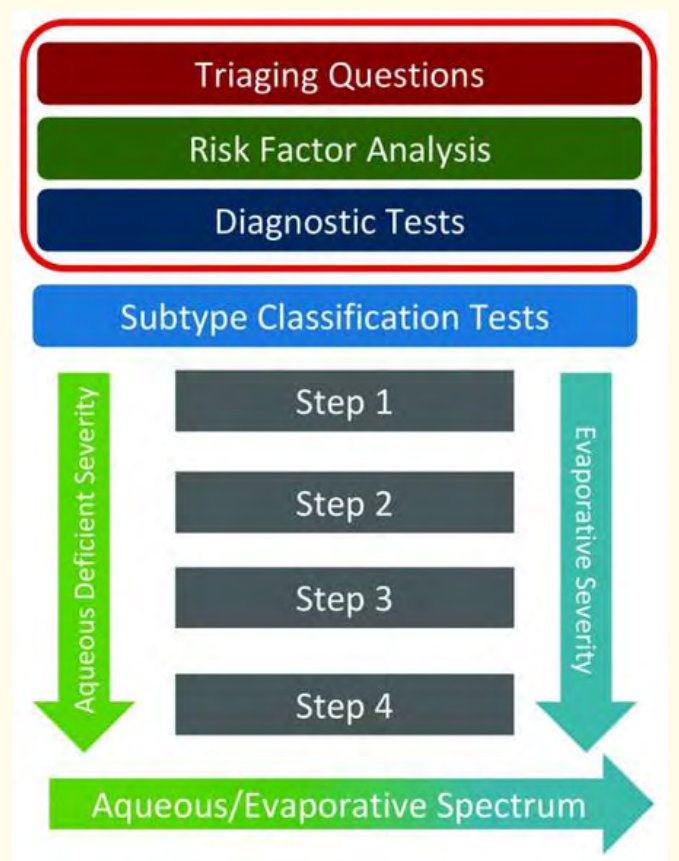

Figure 1: Management of dry eye disease, with key diagnostic steps highlighted [11]. Image courtesy of WebMD Global, LLC.

Using triaging and questionnaires to assess the symptoms of dry eye disease

The TFOS DEWS II report recommends asking patients some triaging questions, which are closed questions. For example, how severe is your dry eye? How is your vision affected? Do your eyes itch? [13]. However, it is also important to ask open questions, as they can be more effective than closed questions. One approach is to record the patient's worst symptom, and then follow this symptom in further examinations. When assessing risk factors, it is very important to ask about smoking, the use of certain medications, contact lens wearing, and other behaviours, because the patient can influence these risk factors. 
The use of questionnaires is also recommended in the TFOS DEWS II report, and they can be useful in everyday clinical practice for diagnosing and assessing dry eye disease [13]. Ocular Surface Disease Index (OSDI) is commonly used for diagnosis, and occasionally for follow up. It can be given to patients in the waiting room before their consultation and provides information on their symptoms and severity of dry eye. The Dry Eye Questionnaire-5 (DEQ-5) questionnaire is also widely used, and a positive score on either one should be followed up with a more detailed examination. Other questionnaires are available, such as Symptom Assessment in Dry Eye (SANDE) which assesses severity and frequency of symptoms and uses a visual analogue scale. SANDE and the Ocular Surface Disease-Quality of Life (OSD-QoL) tend to be used in clinical trials rather than clinical practice $[1,5,15]$.

How far should a specialist go when assessing dry eye disease with a patient who has been referred to them?

In the case of a patient referred for surgery, an assessment of a putative dry eye disease is recommended to mitigate the risks associated with it, such as impaired visual prognosis and patient comfort [16]. Our personal strategy of pre-operative screening is based on three levels of examination, according to the patient's risk of or history of dry eye disease. An assessment is made of the patient's previous history of a range of conditions, procedures and medications, such as dry eye disease, Sjøgren's Syndrome, refractive surgery, antidepressant intake, ocular allergy or anti-glaucoma drugs, and how it was managed. So, for example, a patient with Sjøgren's Syndrome and known dry eye disease or a patient with a history refractive surgery with dry eye disease symptoms, would be considered at high risk for dry eye disease after cataract surgery. Appropriate management of these patients to minimize the risks afterwards (e.g. increasing dry eye disease treatments before surgery, avoiding NSAIDs after surgery, avoiding eye drops containing preservatives such as benzalkonium chloride) is therefore very important. Conversely, in a patient without any known previous history of dry eye disease or risk factors, the assessment can be minimal [16].

\section{Assessing epithelial damage}

Fluorescein staining and lissamine green staining should be used in all patients with suspected disease of the ocular surface system. Fluorescein can provide a lot of information by highlighting damage in the corneal epithelial cells. It also helps with examina- tion of the tear meniscus, for example in assessing the clearance of tears, which is especially important after surgery. Lissamine green is also important because it highlights defects of epithelial cells in the conjunctiva which have been shown to correlate with the level of ocular surface inflammation (see figure 2) [17].

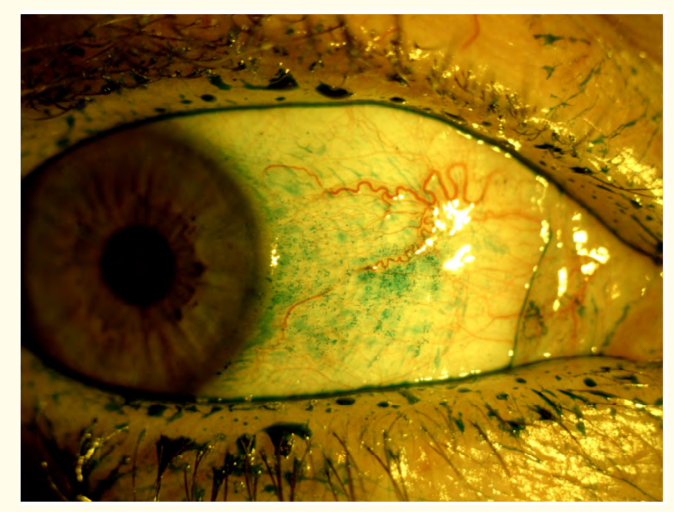

Figure 2: Lissamine green staining of the conjunctiva by using liquid lissamine green (Lissagreen, Polifarma, Italy).

Fluorescein and lissamine green can also be used together, either as a mixture or sequentially. Filters can be used to improve the visibility of staining, a yellow filter for fluorescein and a red filter for lissamine green [14]. Even if these are not mandatory, they increase the contrast and facilitate the assessment of staining in day to day clinical practice.

\section{Assessing tear film stability}

Impaired tear film stability is a key diagnostic measure in dry eye disease. Fluorescein breakup time (FBUT) can be used to assess tear film stability. Studies from Japan have demonstrated that the tear film can break up in different ways. It is important to distinguish between tear film break and thinning. Although both are important, thinning is particularly so $[14,18,19]$. Visual function should be carefully considered in the patient, as tear break up or thinning will lead to a fluctuation in visual quality. It is difficult to define an optimal time for the FBUT measurement, but it could be done 10-30 seconds after installation of fluorescein. It is important to see where there are breaks and what the shape of the break is. Examples of a well-timed FBUT assessments is shown in figure 3, as well as one that was too early and another that was too late. 

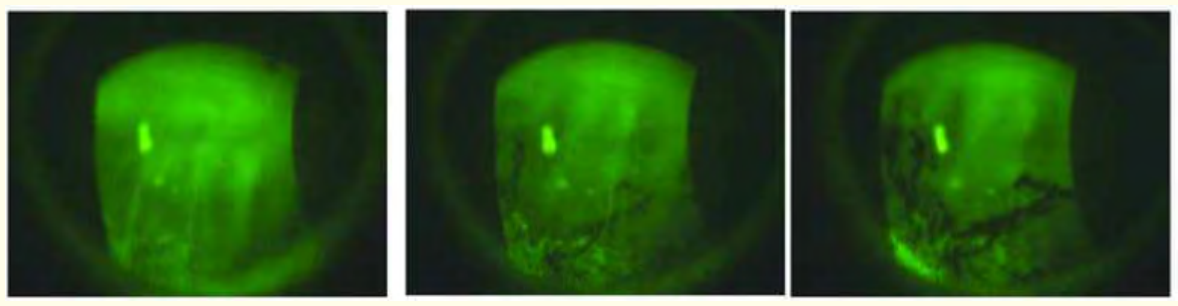

Figure 3: Assessment of the fluorescein breakup time.

Non-invasive breakup time (NBUT) is another way to assess tear film stability. It is an instrument-based assessment that is particularly useful for screening or for assessing a different patient population. But caution is needed because the information provided by NBUT is different from that provided by FBUT. The instrument interprets thinning as a break. It also locates the break and measures the break-up speed. It is helpful to give an objective number and for screening before surgery $[14,18,19]$.

\section{Assessing inflammation in clinical practice}

Inflammation plays an important role in dry eye disease and is a key factor in its pathogenesis [20]. There is probably an acute inflammation phase but it is very brief and is not usually apparent in patients. But inflammation contributes to the chronicity of dry eye disease, and the activation of selected immune factors in the conjunctiva or cornea leads to disease development. The diagnosis and treatment of dry eye disease needs to take inflammation into account.

\section{Tear film osmolarity}

The TFOS DEWS II report proposed assessing tear film osmolarity as an indirect sign of inflammation. Hyperosmolarity of the tear film leads to inflammation and hyperosmolarity increases with the severity of dry eye disease [14].

Matrix metalloproteinase (MMP)-9 in the tear film

Matrix metalloproteinase (MMP)-9 in the tear film is a direct sign of ocular inflammation, and a new MMP-9 immunoassay can be used as a diagnostic tool for dry eye disease [21,22]. The immunoassay has identified the presence of ocular surface inflammation in $40 \%$ of patients with confirmed dry eye disease [21]. A study by Park and colleagues found that patients with dry eye disease who were positive for MMP-9 responded significantly better to anti-inflammatory treatment with cyclosporine than patients who were negative for MMP-9. The study concluded that the MMP-9 immuno- assay may help identify patients with clinically significant ocular surface inflammation who will respond to anti-inflammatory treatment [22].

\section{Assessing the lid margins}

Meibomian gland dysfunction (MGD) is very common in patients with dry eye disease (Figure 4). Morphological changes to the eyelid can be assessed using the slit lamp. Changes to the eyelid margins may include thickening, irregularity and telangiectasia $[14,23]$. In MGD, the meibomian glands are typically blocked. Quantity and quality of meibum secretions from the glands can be assessed using gentle pressure on the eyelid. Normal meibum secretions are clear and easily expressible. In obstructive MGD, the secretions are opaque and thickened, sometimes like toothpaste, and difficult to express from the glands.
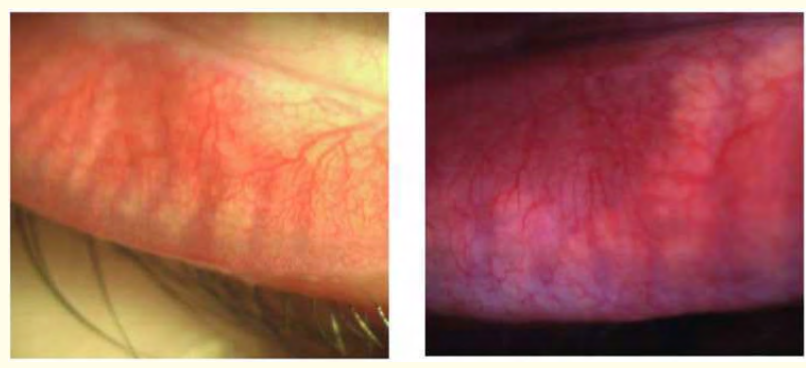

Figure 4: Clinical examination of severe meibomian gland dropout at the slit lamp.

Key recommendations for simplifying the diagnostic process are summarised in table 1.

New technologies for assessing the lipid layer and the meibomian glands

New interferometry-based instruments are available to quantify the tear film lipid layer. They allow automated measurements 
of the thickness of the lipid layer and are therefore helpful in the diagnosis of MGD with a reduced lipid tear film [14].

Meibography is a technique for observing the structure of the Meibomian glands, including the margin and orifices. Traditionally it used white light transillumination, but new techniques are available that use infrared technology.

\begin{tabular}{|c|}
\hline $\begin{array}{c}1 \text { drop of fluorescein for: } \\
\text { Viewing the tear meniscus and film stability } \\
\text { Evaluating TBUT }\end{array}$ \\
Detecting ocular surface damage \\
Can be used alone to diagnose dry eye disease \\
\hline Additional step - lissamine green \\
\hline Schirmer test \\
For diagnosing severe dry eye disease \\
Measuring TMH can be a surrogate of Schirmer test, in terms of \\
aqueous production, if used with caution
\end{tabular}

Table 1: Indications for simplifying the diagnostic process [17].

\section{Potential pitfalls in the assessment of dry eye disease}

There are many potential pitfalls when assessing patients with dry eye disease, but one of the most frequent is when the patient has several clinical signs but very few symptoms. But a patient may also have several symptoms but few clinical signs.

\section{Several signs but very few symptoms}

In a patient who has no symptoms but a lot of ocular surface signs, such as intense ocular surface staining, neurotrophic keratopathy is a differential diagnosis. In these patients it is important to measure corneal sensitivity, to rule out or confirm the disease, because ocular surface irregularities and damage can be the first stage of this devastating disease [14]. Corneal sensitivity can be evaluated using a cotton tip and if the sensation is reduced, the Cochet-Bonnet esthesiometer can be used for a more precise evaluation of the disease, and for patient follow up. The Cochet-Bonnet esthesiometer is very easy to use, though cleaning and maintenance is required, and possible corneal epithelial damage induced.

\section{Several symptoms but very few signs}

Some patients have the opposite situation, with severe symptoms but very few clinical signs. Diagnosis is not easy in these pa- tients, but the best approach is to examine the epithelial cells, because even small amounts of damage can lead to significant pain. As tear film instability can activate nerves, leading to pain, the tear film should also be assessed. Neuropathic pain may be a factor, in which case no damage would be evident. These patients need careful management by the ophthalmologist, as their quality of life may be poor. It is important to distinguish between peripheral and centralized pain. This can be done using a drop of topical anesthetic. The ophthalmologist may need to consider involving a pain specialist in treating the patient.

\section{Conclusions}

Dry eye disease should be considered as a disease of the entire ocular surface system, and all the components of the system need to be taken into account separately, which is not complicated or time-consuming. Dry eye disease is much easier to manage if the examination is made in a systematic and logical way. In the everyday clinical practice fluorescein and lissamine green are very important tool, and with one drop (or better with a calibrated quantity of stain as described by Hamrah., et al. [24]), lot of diagnostic information can be harvested without using any specific technologies. Another easy test that should be included in routine diagnostic processes is corneal sensitivity testing. In most instances this can be performed easily and time-efficiently with a cotton tip applicator.

Although new technologies are not mandatory, they can be very helpful in further exploring the pathophysiological processes of dry eye disease. Moreover, they are an important tool for communicating with patients. For example, showing a patient his/her meibography images with advanced loss of meibomian gland tissue may be of help to explain the need of a chronic treatment much better than with a patient who doesn't have this access to this information.

If the ophthalmologist decides to invest a few minutes in the assessment of his/her patient, underdiagnosis or misdiagnosis of dry eye disease can be minimized. To recognize the association of dry eye and system disease, and not overlook the relationship between the eye and the rest of the body, further specialist consultations (e.g. rheumatologist, internist, allergologist) may be warranted.

\section{Ethics Approval and Consent to Participate}

Not applicable. 


\section{Consent for Publication}

Not applicable.

\section{Availability of Data and Material}

Not applicable.

\section{Competing Interests}

Marc Labetoulle has served as occasional consultant for Alcon, Allergan, Bausch and Lomb, DMG, Dompé, Horus, MSD, Novartis, Santen, Shire, SIFI, Topivert, Thea.

Stefano Barabino has served as occasional consultant for Alcon, Santen, TRB Chemedica, SIFI, Alfa Intes, Polifarma.

Elisabeth Messmer has served as occasional consultant for $\mathrm{Al}$ con Pharma GmbH, DMG, Dompé, Kala, Novartis, Pharm-Allergan GmbH, Santen GmbH, Shire, Sifi, Sun Pharmaceuticals, Théa Pharma GmbH, TRB-Chemedica, Ursapharm Arzneimittel GmbH, Visufarma.

\section{Funding}

Not applicable.

\section{Authors' Contributions}

The authors participated in the educational program on which this article is based, and reviewed and approved the drafts and final version of the manuscript submitted for publication.

\section{Acknowledgements}

Gillian Griffith, BA (Mod), MA, Editor, WebMD Global, LLC, New York, NY, USA, was medical writer for this article, which is based on a Medscape educational program on dry eye disease https://www. medscape.org/viewarticle/922356. Gillian Griffith has no financial or non-financial competing interests to disclose.

\section{Bibliography}

1. Labetoulle M., et al. "Patients' perception of DED and its relation with time to diagnosis and quality of life: an international and multilingual survey". British Journal of Ophthalmology 101 (2017): 1100-1105.

2. Aggarwal S and Galor A. "What's new in dry eye disease diagnosis? Current advances and challenges". F1000 Research 7 (2018): F1000 Faculty Rev-1952.

3. Stapleton F., et al. "TFOS DEWS II epidemiology report". Ocular Surface 15 (2017): 334-365.
4. Ayaki M., et al. "Sleep and mood disorders in dry eye disease and allied irritating ocular diseases". Scientific Reports 6 (2016): 22480.

5. Baudouin C., et al. "Severe impairment of health-related quality of life in patients suffering from ocular surface diseases". Journal Français d'Ophtalmologie 31 (2008): 369-378.

6. Barabino S., et al. "Understanding symptoms and quality of life in patients with dry eye syndrome”. Ocular Surface 14 (2016): 365-376.

7. Labbé A., et al. "Dry eye disease, dry eye symptoms and depression: the Beijing Eye Study". British Journal of Ophthalmology 97 (2013): 1399-1403.

8. Zeev MS., et al. "Diagnosis of dry eye disease and emerging technologies". Clinical Ophthalmology 8 (2014): 581-590.

9. Craig JP., et al. "TFOS DEWS II Definition and Classification Report”. Ocular Surface 15 (2017): 276-283.

10. TFOS-DEWS. "The definition and classification of dry eye disease: report of the Definition and Classification Subcommittee of the International Dry Eye WorkShop". Ocular Surface 5 (2007): 75-92.

11. Jones L., et al. "TFOS DEWS II management and therapy report". Ocular Surface 15 (2017): 802-812.

12. Nichols KK., et al. "The lack of association between signs and symptoms in patients with dry eye disease". Cornea 23 (2004): 762-770.

13. Craig JP., et al. "TFOS DEWS II report executive summary". Ocular Surface 15 (2017): 802-812.

14. Wolffsohn JS., et al. "TFOS DEWS II diagnostic methodology report”. Ocular Surface 15 (2017): 539-574.

15. Shiraishi A and Sakane Y. "Assessment of dry eye symptoms: current trends and issues of dry eye questionnaires in Japan". Investigative Ophthalmology and Visual Science 59 (2018): DES23-DES28.

16. Labetoulle M., et al. "Management of dry eye disease to optimize cataract surgery outcomes: two tables for a daily clinical practice". Journal Français d'Ophtalmologie 2019 42:907-912.

17. Yang S., et al. "The use of conjunctival staining to measure ocular surface inflammation in patients with dry eye". Cornea 38 (2019): 698-705. 
18. Yokoi N and Georgiev GA. "Tear film-oriented diagnosis and tear film-oriented therapy for dry eye based on tear film dynamics". Investigative Ophthalmology and Visual Science 59 (2018): DES13-DES22.

19. Tsubota K., et al. "New perspectives on dry eye definition and diagnosis: a consensus report by the Asia Dry Eye Society". Ocular Surface 15 (2017): 65-76.

20. Baudouin C., et al. "Clinical impact of inflammation in dry eye disease: proceedings of the ODISSEY group meeting". Acta Ophthalmology 96 (2018): 111-119.

21. Messmer EM., et al. "Matrix metalloproteinase 9 testing in dry eye disease using a commercially available point-of-care immunoassay". Ophthalmology 123 (2016): 2300-2308.

22. Park JY., et al. "Matrix metalloproteinase 9 point-of-care immunoassay result predicts response to topical cyclosporine treatment in dry eye disease". Translational Vision Science and Technology 7 (2018): 31.

23. Chhadva P., et al. "Meibomian gland disease: the role of gland dysfunction in dry eye disease". Ophthalmology 124 (2017): S20-S26.

24. Hamrah P., et al. "Optimizing evaluation of lissamine green parameters for ocular surface staining". Eye 25 (2011): 14291434.

\section{Assets from publication with us}

- Prompt Acknowledgement after receiving the article

- Thorough Double blinded peer review

- Rapid Publication

- Issue of Publication Certificate

- High visibility of your Published work

Website: www.actascientific.com/

Submit Article: www.actascientific.com/submission.php

Email us: editor@actascientific.com

Contact us: +919182824667 